\title{
An Improved Distributed Equivalent Circuit Modeling for RF Components by Real-Coefficient AFS Technique
}

\author{
Koon-Tae Kim*, Jae-Hyeong Ko*, Hyun Paek*, Sungtek Kahng** and Hyeong-Seok Kim ${ }^{\dagger}$
}

\begin{abstract}
In this paper, a real-coefficient approach to Adaptive Frequency Sampling (AFS) technique is developed for efficient equivalent circuit modeling of RF components. This proposed method is advantageous than the vector fitting technique and the conventional AFS method in terms of fewer samples leading to a lower order of a rational function on a given data and to a direct conversion to an equivalent circuit for PSPICE(Personal Simulation Program with Integrated Circuit Emphsis) simulation, respectively. To validate the proposed method, the distributed equivalent circuit of a presented multi-layered RF low-pass filter is obtained using the proposed real-coefficient AFS, and then comparisons with EM simulation and circuit simulation for the device under consideration are achieved.
\end{abstract}

Keywords: Distributed equivalent circuit modeling, Adaptive frequency sampling, Real-coefficient AFS, Vector fitting

\section{Introduction}

Modern electronic devices become smaller, while demand for better functions constantly increase. Therefore, the inside of an electronic device, composed of layers of printed circuit boards (PCBs), ends up densely populated. All the components have functions and dimensional efficiencies. However, although artwork is carefully done, the complicated layout may possibly cause EMC (Electromagnetic Compatibiliy) and performance problems [1].

The circuit system consists of an industrial standard number of layers. An electronic system, such as an IED (Intelligent Electronic Device), has eight layers with different layouts stacked. The denser the PCB, the higher is its risk of malfunctioning because apertures between layers and nearby metal lines result to loss and to phase change because of electromagnetic radiation and coupling [2].

To mitigate the aforementioned problems, physical and electrical analyses of an electronic device is strongly needed, which may seem very hard to do because of the complex geometry. Parametric modeling, however, makes things easier. Given a large-scale system in terms of problem solving, it is divided into building blocks, which follow a certain rule. Equivalent circuits or parametric models of all the blocks are found and combined by cascading to give the designer a view of the electrical properties of the entire system [2].

There are a number of approaches in macro modeling. There is a need to first describe frequency response in

$\dagger$ Corresponding Author: School of Electrical and Electronics Eng., Chung-Ang Univ., Korea. (kimcaf2@cau.ac.kr)

* School of Electrical and Electronics Eng., Chung-Ang Univ., Korea.

** Department of Information \& Telecommunication Eng., University of Inchoen, Korea. (s-kahng@inchoen.ac.kr)

Received: October 18, 2010; Accepted: March 9, 2011 simulating or measuring a circuit using a rational function. Although there are conventional rational-function fitting techniques, vector fitting has drawn attention because it gives unconditional stability, which stems from forcing real terms of poles to become negative [3], [4]. Despite the fact that vector fitting gives the fractional terms that directly lead to fixed forms of $R, L$, and $C$, its order is high and is determined by constraints [2]. On the other hand, the Adaptive Frequency Sampling (AFS) technique provides rational function, whose order is more flexible than the sampling order in deciding by using the adaptive process [5], [6]. However, since the coefficients of the numerator and the denominator of the rational function in the existing AFS algorithm are complex, they cannot be converted to equivalent circuits that are done in the vector fitting.

In this paper, we modify the conventional AFS technique by changing its rational function of a given frequencydomain data to one with a real-valued coefficient version, then decomposing it to fractional terms, which has its own equivalent circuits, as in [2]. After which, the resulting distributed equivalent circuit is built in a circuit simulator. Its electrical performance is in good agreement with the original data, with fewer number of elementary circuit blocks. This is presented to validate the proposed scheme by dealing with an RF micro-strip filter with a defected ground structure (DGS), whose data on the electromagnetic field simulation are provided.

\section{Conventional and Proposed AFS}

Given $H(s)$ as a frequency response of an RF component, it can be expressed as follows: 


$$
H(s)=\frac{a_{0}+a_{1} s+a_{2} s^{2}+\ldots . .+a_{q} s^{q}}{1+b_{0} s+b_{1} s^{2}+b_{2} s^{3}+\ldots . .+b_{p} s^{p+1}}=\frac{\sum_{k=0}^{q} a_{k} s^{k}}{1+\sum_{k=0}^{p} b_{k} s^{k+1}}
$$

where $s$ and $j$ mean $j \omega$ and $\sqrt{-1}$, respectively. The AFS algorithm to get Eq. (1) is depicted in the following chart:

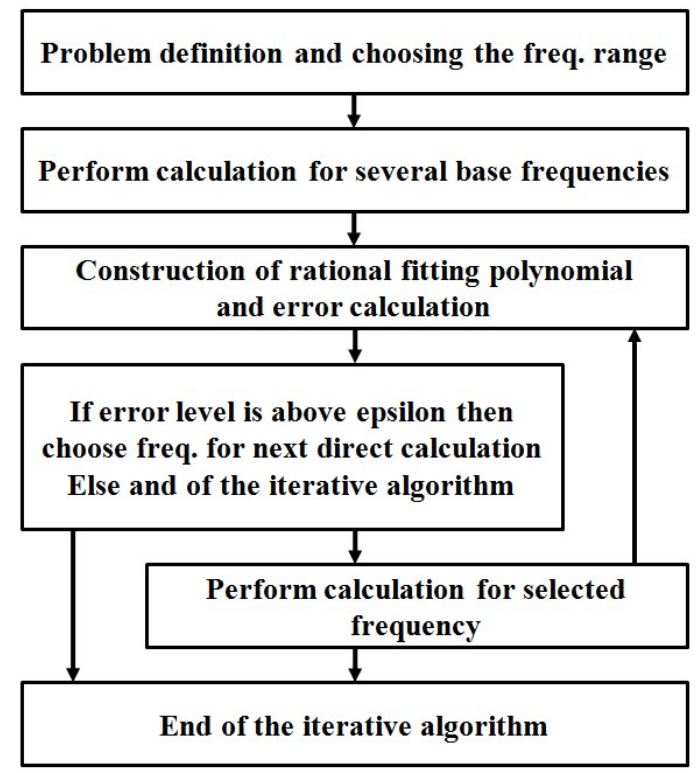

Fig. 1. Adaptive Frequency Sampling (AFS) algorithm

Starting with the smallest number of samples from the given data, it undergoes the iterative steps of estimating error, progressing to spotting the largest error point from the former stage and including it at a later stage to minimize total error.

As for a rational function, there are numerous equivalent circuit expressions. If we decompose them to the fractional terms of real-coefficients as practiced in [2], they turn into the forms of $R, L$, and $C$, following the relations introduced in [2]. Checking the conventional AFS in [5], the resultant forms have only complex coefficients which are not suitable to the conversion method. As such, we propose an efficient scheme to obtain the parametric model, which has the benefits of vector fitting's easy conversion to equivalent circuits and to lower-ordered sampling of the AFS. This is enabled by splitting Eq. (1) into real and imaginary parts and equating them with their counterparts in the samples [7].

$$
\begin{aligned}
\sum_{k=0}^{p} b_{k} s^{k+1} & =j \sum_{k=0}^{p / 2} b_{2 k}(-1)^{k} \omega^{2 k+1}+\sum_{k=1}^{p / 2} b_{2 k-1}(-1)^{k} \omega^{2 k} \\
\sum_{k=0}^{q} a_{k} s^{k} & =\sum_{k=0}^{q / 2} a_{2 k}(-1)^{k} \omega^{2 k}-j \sum_{k=1}^{q / 2} a_{2 k-1}(-1)^{k} \omega^{2 k-1}
\end{aligned}
$$

$$
\begin{aligned}
H_{R}(\omega)= & \left\{H_{I}(\omega) \sum_{k=0}^{p / 2} b_{2 k}(-1)^{k} \omega^{2 k+1}-H_{R}(\omega) \sum_{k=1}^{p / 2} b_{2 k-1}(-1)^{k} \omega^{2 k}\right\} \\
& +\sum_{k=0}^{q / 2} a_{2 k}(-1)^{k} \omega^{2 k} \\
H_{I}(\omega)= & -\left\{H_{R}(\omega) \sum_{k=0}^{p / 2} b_{2 k}(-1)^{k} \omega^{2 k+1}+H_{I}(\omega) \sum_{k=1}^{p / 2} b_{2 k-1}(-1)^{k} \omega^{2 k}\right\} \\
& -\sum_{k=1}^{q / 2} a_{2 k-1}(-1)^{k} \omega^{2 k-1}
\end{aligned}
$$

Subscripts $R$ and $I$ represent the real and the imaginary parts, respectively. Once real-coefficients $a_{k}$ and $b_{k}$ are found, the rational function is broken down into 1st- and 2nd-order fractional terms, as shown in Fig. 2. If $H(s)$ is an admittance function $\mathrm{Y}(\mathrm{s})$, it is simply expressed by the sum of the sub-admittance corresponding to its fractional terms.

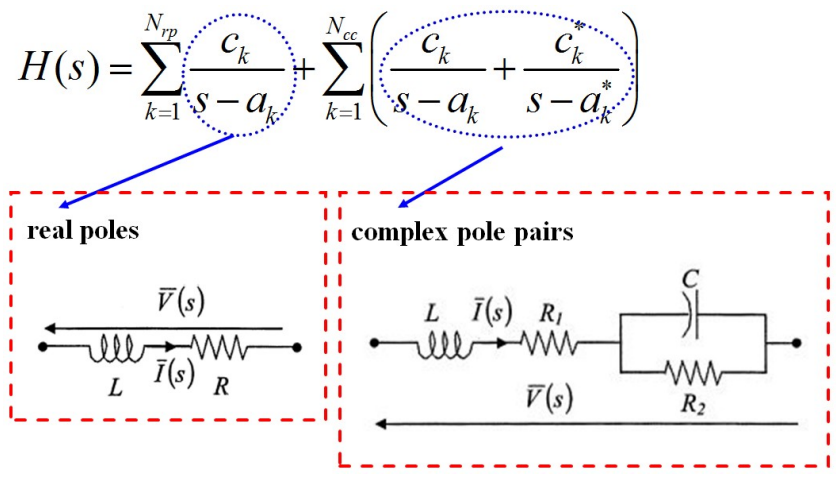

Fig. 2. The proposed AFS expressed as a circuit

\section{Equivalent Circuit Modeling by the Proposed AFS}

In this section, we show the advantages of the proposed AFS over the standard vector fitting technique and the conventional AFS method by testing two examples and finding their equivalent circuits. First, a commonly used micro-strip low-pass filter is used to compare the vector fitting technique and the proposed AFS method from the standpoint of order and accuracy. Before describing the low-pass filter for this test, the basic configuration of the equivalent circuit is introduced. There are several ways to express a circuit equivalent to the measured or the simulated result of the real device or circuit, say, admittance or impedance. Also, for the admittance expression, the configuration can be the $\pi$-network or the T-network. When the measurement or simulation data and the admittance are given, they can be directly converted to the $\pi$-network, including the set of elements in Fig. 3, by vector fitting or by the proposed AFS.

As shown in Fig. 3, admittance is broken down into $Y_{1}$, $\mathrm{Y}_{2}$, and $\mathrm{Y}_{3}$ elements. Each of these elements consists of a sub-admittance. Our example is a micro-strip low-pass 


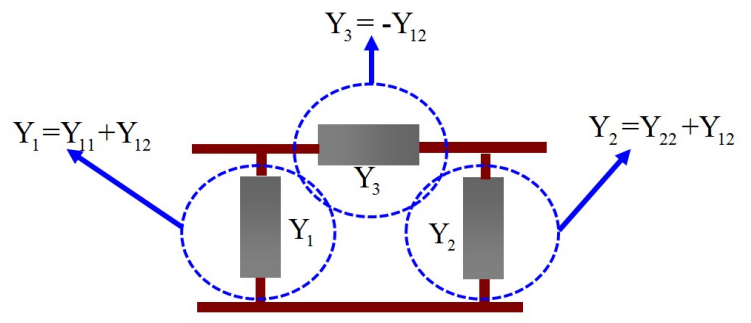

(a)

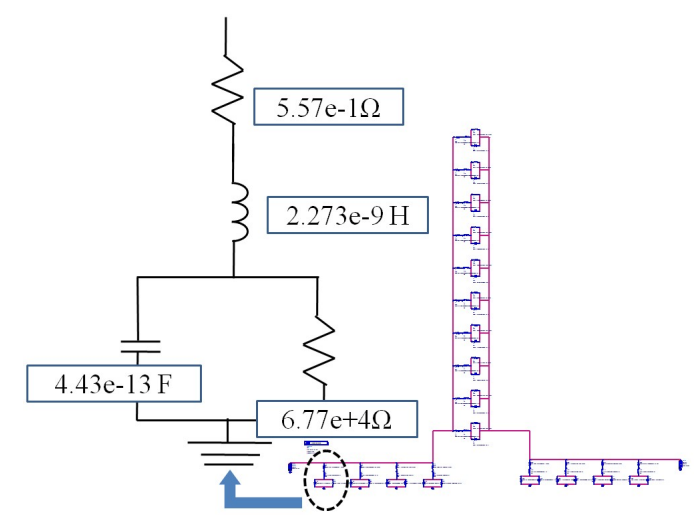

(b)

Fig. 3. The $\pi$-network, including its set of elements; (a) $\pi$ network and (b) representative elements

filter with alternating impedance steps. Data are obtained by CST-MWS(CST-Microwave Studio) simulation, which is a popular and reliable $3 \mathrm{D}$ EM solver.

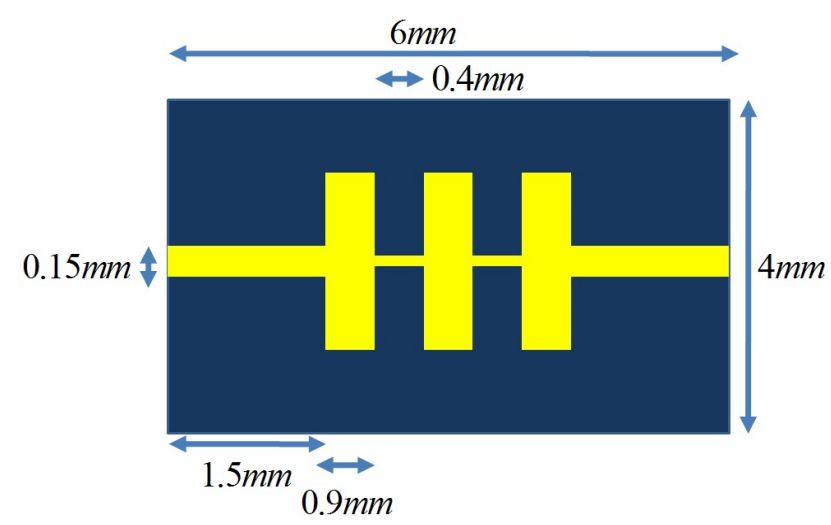

Fig. 4. A typical micro-strip low-pass filter

The top view of the micro-strip filter appears with information on its physical dimensions. Considering that the performance of low-pass filters is addressed a lot in many textbooks, we try to focus on the similarities and the differences between vector fitting and the proposed AFS.

In Table 1, the two techniques are compared while being used to express the CST-MWS simulated results of the example above, in the form of $\pi$-network admittance as rational functions, to have perfect agreement with the simulation data. In Table 1, the numbers in the second and
Table 1. Comparisons between vector fitting and the proposed AFS

\begin{tabular}{c|c|c}
\hline & Vector fitting & AFS \\
\hline Error of $\mathrm{Y}_{1}$ & $3.21 \mathrm{e}-4$ & $1.03 \mathrm{e}-4$ \\
\hline Error of $\mathrm{Y}_{3}$ & $1.21 \mathrm{e}-4$ & $1.40 \mathrm{e}-4$ \\
\hline Number of poles for $\mathrm{Y}_{1}$ & 12 & 5 \\
\hline Number of poles for $\mathrm{Y}_{3}$ & 14 & 11 \\
\hline
\end{tabular}

in the third columns represent the errors between the simulation data and vector fitting, and the errors between the simulation data and the proposed AFS over the sample points, respectively. Vector fitting and the proposed AFS both show ignorable errors in the simulation data. The proposed AFS method requires lower-order rational functions (fewer poles) than vector fitting, which needs $\mathrm{Y}_{1}$ poles of 12 and $\mathrm{Y}_{3}$ poles of 14 for the same accuracy. In other words, the proposed AFS method takes fewer sample points from the original data than vector fitting. Moreover, the fewer the numbers of poles, the fewer the number of elementary circuit blocks is produced in constituting the whole distributed equivalent circuit than the vector fitting. This shows the efficiency of the proposed AFS in the parametric modeling of RF components.

The proposed AFS is superior to conventional AFS [8]. Actually, conventional AFS cannot possibly convert its rational data function into an equivalent circuit that is treated in PSPICE. Only the proposed AFS can change its own rational function to a Spice-type circuit. Thus, although we explicitly compare the equivalent circuits between the two methods, their different complexities in the coefficients of their rational functions are observed. Also, we examine the accuracy of the proposed AFS for simulated data. The geometry we use in comparing conventional AFS and the proposed AFS method is a low-pass filter we have designed, which adopts a DGS, because it can change the characteristics of a transmission line, such as line capacitance and inductance in [8]. This is more complicated than the first example because multi-layer considerations are needed and results in size reduction in low-pass filter design practices. Fig. 5 shows a relatively simple DGS low-pass filter for obtaining coefficients with comparative analysis between the conventional and the proposed methods.

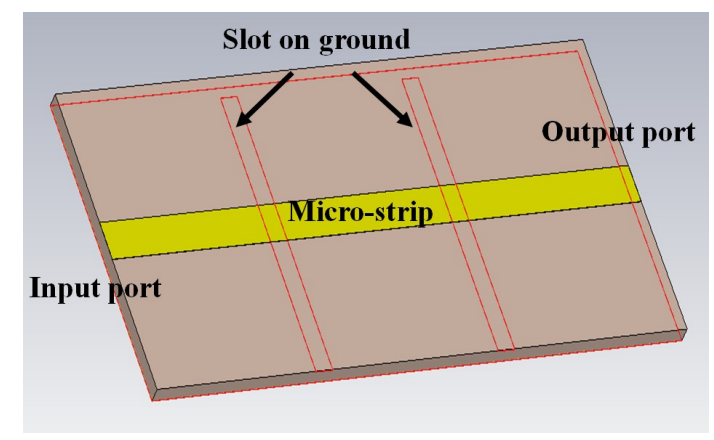

Fig. 5. DGS microstrip low-pass filter for comparison 


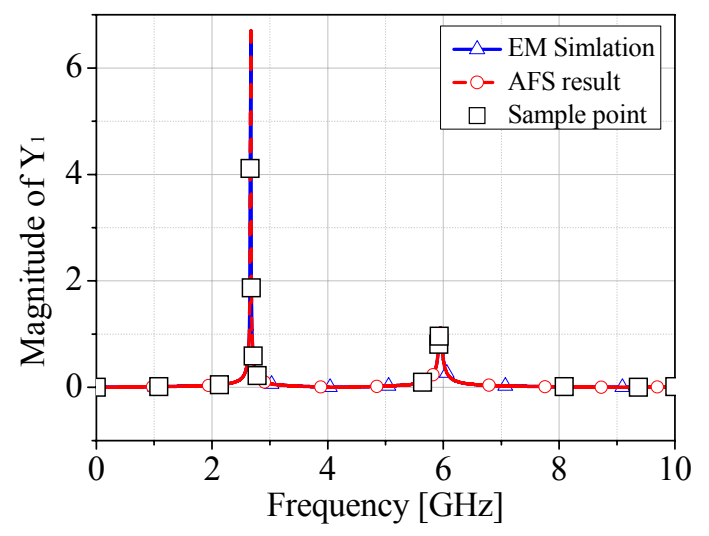

(a)

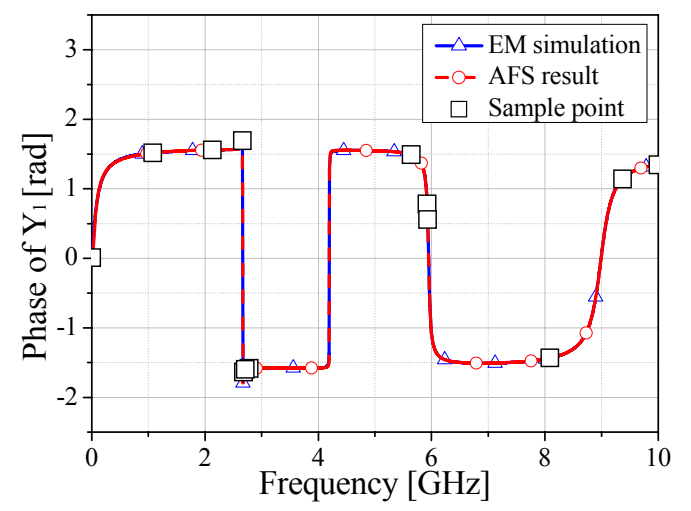

(b)

Fig. 6. $Y_{1}$ rational function of the proposed AFS; (a) Magnitude and (b) Phase

The micro-strip under simulation has a metal strip on a $0.017 \mathrm{~mm}$ thick substrate of $\varepsilon_{\mathrm{r}}=3$ and a horn-shaped slot on the ground. The $\mathrm{Y}_{1}$-part of the $\pi$-parametric model above is computed using conventional AFS ( $\mathrm{Y}_{2}$ and $\mathrm{Y}_{3}$ can be calculated similarly). Owing to the fact that AFS needs fewer samples compared with vector fitting, the resultant rational function $\mathrm{Y}_{1}\left(=\mathrm{Y}_{11}+\mathrm{Y}_{12}\right)$ fits the phase and the magnitude of a given data with only 19 samples. However, they have tabulated complex values $a_{k}$ and $b_{k}$. Thus, the circuit elements for the $\pi$-parametric model cannot be obtained.

However, through the scheme, real values $a_{k}$ and $b_{k}$ are calculated by following the procedures presented in Eqs. (2) and (3), which make the following equivalent circuit whose $\mathrm{S}_{21}$ data fit the original data.

This paper emphasizes that the proposed AFS can make a rational function only with real-coefficients, which can be implemented with parametric equivalent circuit blocks. Fig. 7 shows comparisons of transmission performances between the EM and the mathematical simulations, with the rational function of complex coefficients shown in Table 2. Even though Fig. 7 shows excellent agreements both in the observation of the magnitude and in the phase, rational function with complex coefficients cannot be implemented with parametric equivalent circuit blocks.

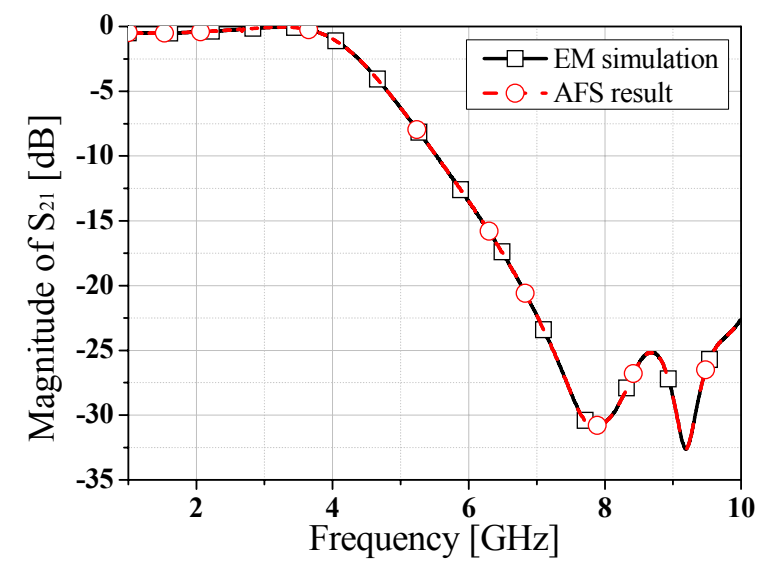

(a)

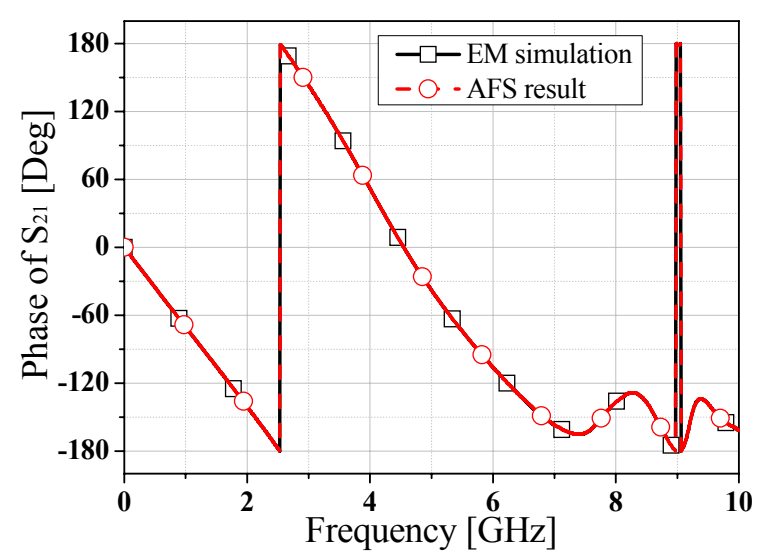

(b)

Fig. 7. S-parameter of the proposed AFS; (a) Magnitude and (b) Phase

Table 2. Complex $a_{k}$ and $b_{k}$ by conventional AFS

\begin{tabular}{c|c|c}
\hline $\mathrm{K}$ & Denominator & Numerator \\
\hline 0 & $1.00 \mathrm{e}+000$ & $-1.02 \mathrm{e}+004-2.72 \mathrm{e}+004 \mathrm{i}$ \\
\hline 1 & $-1.08 \mathrm{e}+002-3.21 \mathrm{e}+001 \mathrm{i}$ & $-8.48 \mathrm{e}-002-7.79 \mathrm{e}-004 \mathrm{i}$ \\
\hline 2 & $1.94 \mathrm{e}-007+7.04 \mathrm{e}-008 \mathrm{i}$ & $4.30 \mathrm{e}-010-1.19 \mathrm{e}-009 \mathrm{i}$ \\
\hline 3 & $-1.02 \mathrm{e}-016-6.85 \mathrm{e}-017 \mathrm{i}$ & $-5.96 \mathrm{e}-019+2.28 \mathrm{e}-018 \mathrm{i}$ \\
\hline 4 & $-2.35 \mathrm{e}-026+4.18 \mathrm{e}-026 \mathrm{i}$ & $3.58 \mathrm{e}-028-1.70 \mathrm{e}-027 \mathrm{i}$ \\
\hline 5 & $5.27 \mathrm{e}-035-1.99 \mathrm{e}-035 \mathrm{i}$ & $-9.27 \mathrm{e}-038+5.58 \mathrm{e}-037 \mathrm{i}$ \\
\hline 6 & $-2.82 \mathrm{e}-044+8.16 \mathrm{e}-045 \mathrm{i}$ & $3.31 \mathrm{e}-048-2.65 \mathrm{e}-047 \mathrm{i}$ \\
\hline 7 & $8.33 \mathrm{e}-054-2.66 \mathrm{e}-054 \mathrm{i}$ & $1.15 \mathrm{e}-057-4.01 \mathrm{e}-056 \mathrm{i}$ \\
\hline 8 & $-1.57 \mathrm{e}-063+6.14 \mathrm{e}-064 \mathrm{i}$ & $1.29 \mathrm{e}-066+1.45 \mathrm{e}-065 \mathrm{i}$ \\
\hline 9 & $2.03 \mathrm{e}-073-9.30 \mathrm{e}-074 \mathrm{i}$ & $-6.76 \mathrm{e}-076-2.60 \mathrm{e}-075 \mathrm{i}$ \\
\hline 10 & $-1.82 \mathrm{e}-083+8.61 \mathrm{e}-084 \mathrm{i}$ & $1.36 \mathrm{e}-085+2.99 \mathrm{e}-085 \mathrm{i}$ \\
\hline 11 & $1.03 \mathrm{e}-093-4.39 \mathrm{e}-094 \mathrm{i}$ & $-1.42 \mathrm{e}-095-2.37 \mathrm{e}-095 \mathrm{i}$ \\
\hline 12 & $-2.66 \mathrm{e}-104+9.53 \mathrm{e}-105 \mathrm{i}$ & $7.64 \mathrm{e}-106+1.16 \mathrm{e}-105 \mathrm{i}$ \\
\hline 13 & & $-1.72 \mathrm{e}-116-2.41 \mathrm{e}-116 \mathrm{i}$ \\
\hline
\end{tabular}

However, the coefficients for rational function shown in Table 3 are totally different from those of the conventional AFS method, which end up with complex coefficients (Table 2). The proposed AFS has real-coefficients in the numerator and in the denominator of its own rational function. 


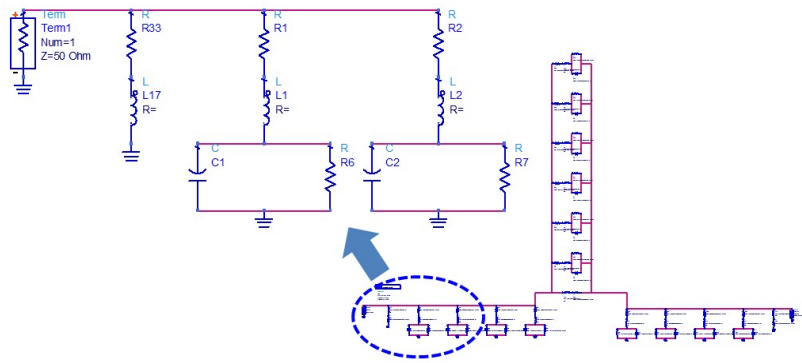

(a)

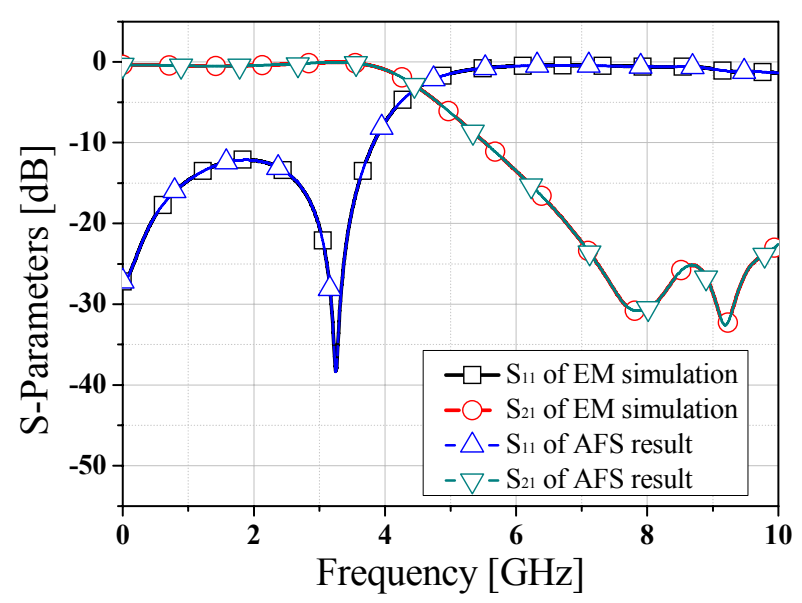

(b)

Fig. 8. Design of equivalent circuit model; (a) Equivalent circuit of low-pass filter and (b) Comparison between EM simulation and AFS

Table 3. Real $a_{k}$ and $b_{k}$ by the proposed AFS

\begin{tabular}{c|c|c}
\hline $\mathrm{K}$ & Denominator & Numerator \\
\hline 0 & $1.00 \mathrm{e}+000$ & $8.76 \mathrm{e}-004$ \\
\hline 1 & $-6.97 \mathrm{e}-010$ & $9.65 \mathrm{e}-013$ \\
\hline 2 & $-2.32 \mathrm{e}-019$ & $-1.28 \mathrm{e}-021$ \\
\hline 3 & $-2.81 \mathrm{e}-029$ & $-4.04 \mathrm{e}-031$ \\
\hline 4 & $-2.57 \mathrm{e}-039$ & $-4.40 \mathrm{e}-041$ \\
\hline 5 & $-1.12 \mathrm{e}-049$ & $-3.30 \mathrm{e}-051$ \\
\hline 6 & $-7.60 \mathrm{e}-060$ & $-7.32 \mathrm{e}-062$ \\
\hline 7 & $-7.36 \mathrm{e}-071$ & $-4.97 \mathrm{e}-072$ \\
\hline 8 & $-5.27 \mathrm{e}-081$ & $-1.43 \mathrm{e}-083$ \\
\hline 9 & $2.45 \mathrm{e}-093$ & $-1.61 \mathrm{e}-093$ \\
\hline 10 & $-7.82 \mathrm{e}-103$ & $6.30 \mathrm{e}-105$ \\
\hline 11 & $6.04 \mathrm{e}-114$ & $-1.15 \mathrm{e}-115$ \\
\hline 12 & $-1.32 \mathrm{e}-125$ & $1.44 \mathrm{e}-126$ \\
\hline 13 & $5.61 \mathrm{e}-136$ & \\
\hline
\end{tabular}

As shown in Table 3, because the proposed AFS has real-coefficients, it has less complexity compared with conventional AFS. Fig. 8(a) shows the PSPICE-wise distributed equivalent circuit expressed by the obtained real-coefficients. Fig. 8(b) shows the full comparison of Sparameters between the full 3D-EM and the circuit simulations with the distributed equivalent circuit obtained using the proposed AFS. The ADS(Advanced Design System) simulation results with the distributed equivalent circuit obtained using the proposed technique also show excellent agreements both in transmission and in the reflection of 3D-EM simulation.

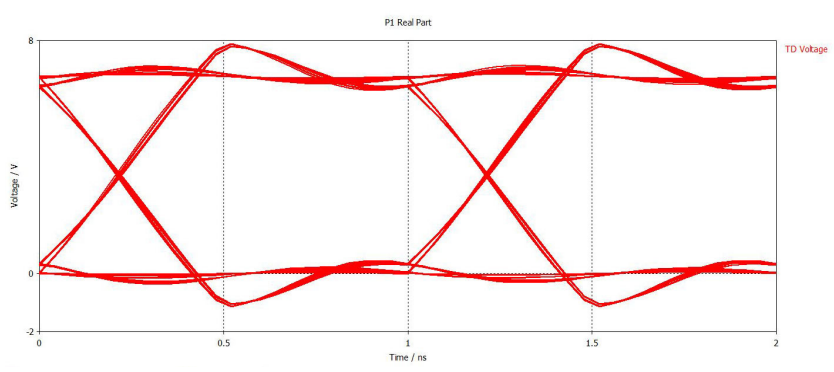

(a)

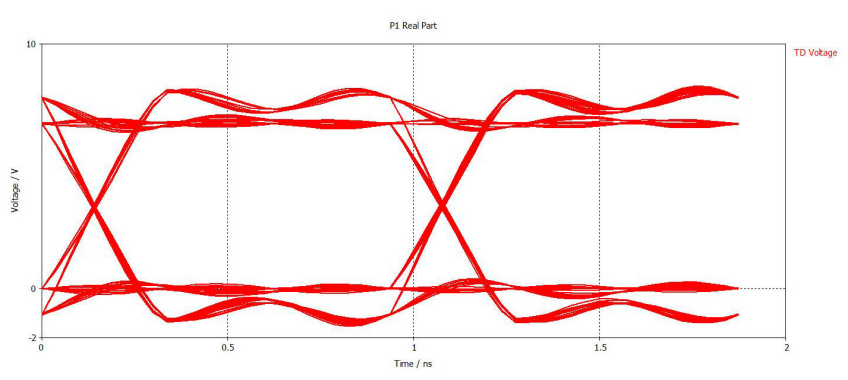

(b)

Fig. 9. Eye diagram performance comparison; (a) 3D-EM simulation and (b) equivalent circuit simulation

Further, the time-domain performance of the RF components is frequently required in the delay and noise anlaysis of various circuit and system designs with digital broadband signals. Fig. 9 shows the eye diagram with pulse train excitations of the 3D-EM simulation results and the distributed equivalent circuit obtained using the proposed method. As shown in the comparison, the time-domain performace of the proposed method's circuit also provides enough accuracy for the design requirements.

\section{Conclusion}

We proposed an efficient rational function fitting method for obtaining useful distributed equivalent circuit with frequency response results using real-coefficient AFS method. To prove the validity of the proposed method, we took account of RF low-pass filters under comparison. The various comparisons showed enough validity for this paper, with usefulness both in frequency and time-domain approaches.

\section{Acknowledgment}

This work was supported by KESRI (2009T100100596), which is funded by the Ministry of Knowledge Economy (MKE). 


\section{References}

[1] Kwang Lim Choi, Nanju Na, and Madhavan Swaminathan, "Characterization of embedded passives using macromodels in LTCC technology", IEEE Transactions Vol 21, No. 3, August 1998.

[2] G. Antonini, "SPICE Equivalent Circuit of Frequency-Domain Responses", IEEE Trans. on EMC, vol. 45, no.3, pp.502-512, Aug. 2003.

[3] Bjorn Gustavsen "Rational Approximation of Frequency Domain Reponses by Vector Fitting", IEEE Transactions on Power Delivery, Vol. 14, No. 3, July 1999.

[4] S. Kahng, "Prediction of the Radiated Emission(RE)s due to the PCB Power-Bus' Resonance Modes and Mitigation of the RE Levels," Journal of the Korea Electromagnetic Engineering Society, Vol. 7, No. 1, pp.7-11, March, 2007.

[5] S. Song, "Frequency Domain Analysis of Microstrip Filters and Antennae Using an AFS MoM", IEEE Trans. on Magnetics Vol 42, No 4, April 2006.

[6] Seunghyun Song, Hyeong-Seok Kim, Hyun-kyo Jung, Jeffrey Braunstein and Un-chul Moon, "Frequency domain analysis of microstrip filters and antennae using and adaptive frequency sampling moment method" IEEE Transactions on Magnetics, Vol. 42, No. 4, Apri 2006.

[7] Yonungwook Kim and Hao King, "Equivalent circuit modeling of broadband antennas using a rational function approximation", Department of Electrical and Computer engineering, The University of Texas at Austin, Nov 2005.

[8] D. Ahn, J.-S. Park, C.-S. Kim, J. Kim, Y. Qian, T. Itoh, "A design of the low-pass filter using the novel microstrip defected ground structure" IEEE Trans. On MTT, Vol. 49, No. 1, pp.86-93, January 2001.

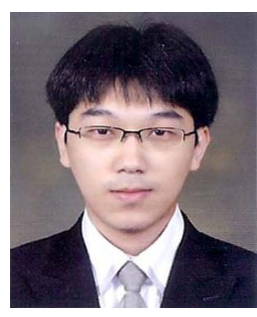

Koon-Tae Kim was born in Jeju, South Korea. He received his B.S. degree in Information Telecommunications from Seo-Kyeong University and his M.S. degree in electrical and electronics engineering from Chung-Ang University, South Korea in 2008 and 2010 , respectively. He is working toward a $\mathrm{Ph} . \mathrm{D}$ degree in electrical and electronics engineering at Chung-Ang University, South Korea. His interests include optimizing designs of passive components and equivalent circuit models, and analysis of RF components.

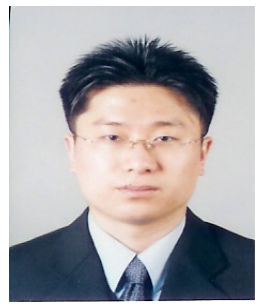

Jae-Hyeong Ko was born in Jeju, South Korea. He received his B.S. and M.S. degrees from Chung-Ang University, South Korea in 2004 and 2008, respectively. He is currently working toward a Ph.D. degree in electrical and electronics engineering at Chung-Ang University, South Korea. His interests include studies in microwave circuits, antenna, optimal design, and robust design of passive components.

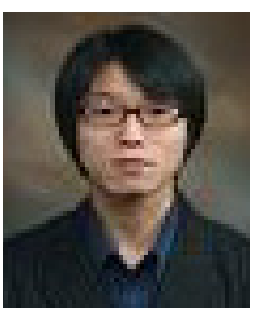

Hyun Paek was born in Seoul, South Korea. He received his B.S. and M.S. degrees in electrical and electronics engineering from Chung-Ang University, South Korea in 2008 and 2010, respectively. His interests include equivalent circuit modeling, and analysis and design of microwave passive

components.

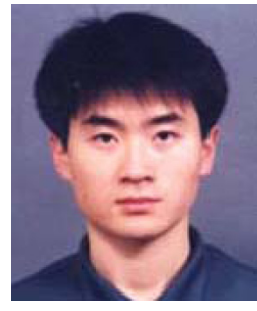

Sungtek Kahng was born in Seoul, South Korea. He received his Ph.D. degree in electronics and communications engineering, specializing in radio science and engineering, from Hanyang University, South Korea in 2000. From 2000 to early 2004, he worked for the Electronics and Telecommunication Research Institute, where he worked on numerical electromagnetic characterization of and developed RF passive components for satellites. Since March 2004, he has joined the Department of Information and Telecommunication Engineering at the University of Incheon, where he continues to research on analysis and advanced design methods of microwave components and antennas, including metamaterial technologies and wireless power transfer. $\mathrm{He}$ is also included in the Science \& Engineering of Marquis' Who's Who in the World and holds several patents on EMC solutions and on microwave and millimeter-wave components as well.

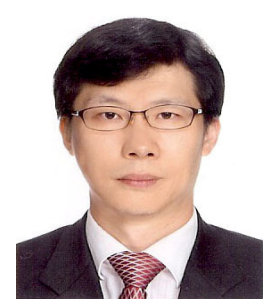

Hyeong-Seok Kim was born in Seoul, South Korea on October 9, 1962. He received his B.S., M.S., and Ph.D. degrees from the Department of Electrical Engineering at Seoul National University, Seoul, South Korea in 1985, 1987, and 1990, respectively. From 1990 to 2002, he was with the Division of Information Technology Engineering, Soonchunhyang University, Asan, South Korea. In 1997, he was a visiting professor at the Electrical Computer Science Engineering, Rensselaer Polytechnic Institute, Troy, New York, USA. In 2002, he transferred to the School of Electrical and Electronics Engineering, Chung-Ang University, Seoul, South Korea as full professor. His current research interests include numerical analysis of electromagnetic fields and waves, analysis and optimal design of passive and active components for wireless communications, RFID applications, power information technology, and electromagnetic education. He is the corresponding author in this paper. 